\title{
LIE AUTOMORPHISMS OF INCIDENCE ALGEBRAS
}

\author{
ÉRICA Z. FORNAROLI, MYKOLA KHRYPCHENKO, AND EDNEI A. SANTULO JR.
}

\begin{abstract}
Let $X$ be a finite connected poset and $K$ a field. We give a full description of the Lie automorphisms of the incidence algebra $I(X, K)$. In particular, we show that they are in general not proper.
\end{abstract}

\section{INTRODUCTION}

Let $A$ be an associative algebra over a commutative ring $R$. Then $A$ becomes a Lie algebra under the commutator $[a, b]=a b-b a$ of $a, b \in A$. By a Lie automorphism of $A$ one means an automorphism of the Lie algebra $(A,[]$,$) . Clearly, an$ automorphism $\varphi$ of $A$ and the negative $-\psi$ of an anti-automorphism $\psi$ of $A$ are Lie automorphisms of $A$. Moreover, if $\nu$ is an $R$-linear central-valued map on $A$ such that $\nu([A, A])=\{0\}$ and $\phi$ is either $\varphi$ or $-\psi$ as above, then $\phi+\nu$ is a Lie endomorphism of $A$, which, under certain assumptions on $\nu$, becomes bijective. We will call such Lie automorphisms proper. Hua [9] proved that each Lie automorphism of the full matrix ring $M_{n}(R), n>2$, over a division $\operatorname{ring} R$, $\operatorname{char}(R) \notin\{2,3\}$, is proper. Martindale extended this result to Lie isomorphisms between primitive rings [13], simple rings [15] and, yet more generally, between prime rings [14]. Đoković [7] described the group of Lie automorphisms of the upper triangular matrix algebra $T_{n}(R)$, where $R$ is a commutative unital ring with trivial idempotents. It follows from his description that each Lie automorphisms of $T_{n}(R)$ is proper (see Corollary 5.19). Cao [5] generalized the result by Đoković to the case of commutative rings without any restriction on idempotents. A similar result has also been independently proved by Marcoux and Sourour [12]. Cecil [6] showed that Lie isomorphisms between block-triangular matrix algebras over a UFD are proper.

The incidence algebra $I(X, R)$ of a locally finite poset $X$ over a commutative unital ring $R$ is a natural generalization of $T_{n}(R)$. Jordan and Lie maps on $I(X, R)$ (and even on more general algebras) have been actively studied during the last 5 years (see [1, 2, 3, 11, 17, 18, 19]). Usually, all Lie-type maps on $I(X, R)$ are proper. This is no longer the case for Lie automorphisms of $I(X, K)$, where $K$ is a field and $X$ is finite and connected, as we show in this paper. In Section 1 we recall basic definitions about posets and their incidence algebras. In Sections 2 and 3 we prove several facts on the structure of $(I(X, K),[]$,$) which will be used throughout the$ work. In Section 4 we reduce the description of all Lie automorphisms of $I(X, K)$ to the description of those which we call elementary (see Theorem 4.15). Section 5 is the main part of the paper. We describe elementary Lie automorphisms of $I(X, K)$ in terms of triples $(\theta, \sigma, c)$, where $\theta$ is a bijection of $B=\left\{e_{x y}: x<y\right\}$, monotone on maximal chains in $X$ and satisfying a certain combinatorial condition which

2010 Mathematics Subject Classification. Primary: 16S50, 17B60, 17B40; secondary: 16W10.

Key words and phrases. Lie automorphism, incidence algebra, automorphism, antiautomorphism. 
involves cycles in $X, \sigma$ is a "1-cocycle-looking" map related to $\theta$ and $c$ is a sequence of $|X|$ elements of $K$ such that $\sum c_{i} \neq 0$ (see Theorem 5.18).

\section{Preliminaries}

1.1. Posets. Let $(X, \leq)$ be a partially ordered set (poset, for short) and let $x, y \in$ $X$. The interval from $x$ to $y$ will be denoted by $\lfloor x, y\rfloor$, that is, $\lfloor x, y\rfloor=\{z \in X$ : $x \leq z \leq y\}$. If all the intervals of $X$ are finite, then $X$ is said to be locally finite. A chain in $X$ is a linearly ordered (under $\leq$ ) subset of $X$. The length of a finite chain $C \subseteq X$ is $|C|-1$. More generally, the length of a finite non-empty subset $Y \subseteq X$, denoted by $l(Y)$, is the maximum length of chains $C \subseteq Y$. Obviously, $l(Y) \leq|Y|-1$. A walk in $X$ is a sequence $x_{0}, x_{1}, \ldots, x_{m}$ of elements of $X$, such that $x_{i}$ is comparable with $x_{i+1}$ and, moreover, $l\left(\left\lfloor x_{i}, x_{i+1}\right\rfloor\right)=1$ (if $x_{i} \leq x_{i+1}$ ) or $l\left(\left\lfloor x_{i+1}, x_{i}\right\rfloor\right)=1$ (if $\left.x_{i+1} \leq x_{i}\right)$ for all $i=0, \ldots, m-1$. A walk $x_{0}, x_{1}, \ldots, x_{m}$ is said to be closed if $x_{0}=x_{m}$. A path is a walk in which $x_{i} \neq x_{j}$ for $i \neq j$. A cycle is a closed walk $x_{0}, x_{1}, \ldots, x_{m}=x_{0}$ such that $m \geq 4$ and $x_{i}=x_{j} \Rightarrow\{i, j\}=\{0, m\}$ for $i \neq j$. The set $X$ is said to be connected if for any pair of $x, y \in X$ there is a path $x=x_{0}, \ldots, x_{m}=y$. In this work $X$ will always be a connected finite poset.

1.2. Incidence algebras. Let $X$ be a locally finite poset and let $K$ be a field. The incidence algebra $I(X, K)$ of $X$ over $K$ is the set $I(X, K)=\{f: X \times X \rightarrow K$ : $f(x, y)=0$ if $x \not \leq y\}$ endowed with the usual structure of a vector space over $K$ and the product defined by

$$
(f g)(x, y)=\sum_{x \leq t \leq y} f(x, t) g(t, y)
$$

for any $f, g \in I(X, K)$. Then $I(X, K)$ is a $K$-algebra with identity $\delta$ given by

$$
\delta(x, y)= \begin{cases}1, & x=y \\ 0, & x \neq y\end{cases}
$$

Moreover, if $X$ is finite, which is the case we deal with in this paper, $I(X, K)$ admits the standard basis $\left\{e_{x y}: x \leq y\right\}$, where

$$
e_{x y}(u, v)= \begin{cases}1, & (u, v)=(x, y), \\ 0, & (u, v) \neq(x, y) .\end{cases}
$$

Indeed, $f=\sum_{x \leq y} f(x, y) e_{x y}$ for all $f \in I(X, K)$. We will denote $e_{x}=e_{x x}$.

Let $B=\left\{e_{x y}: x<y\right\}$. It is known (see [16, Theorem 4.2.5]) that the Jacobson radical of $I(X, K)$ is

$$
J(I(X, K))=\{f \in I(X, K): f(x, x)=0 \text { for all } x \in X\}=\operatorname{Span}_{K} B .
$$

An element $f \in I(X, K)$ is said to be diagonal, if $f(x, y)=0$ for $x \neq y$. Diagonal elements form a commutative subalgebra of $I(X, K)$ spanned by $\left\{e_{x}: x \in X\right\}$, which we denote by $D(X, K)$. Clearly, each $f \in I(X, K)$ can be uniquely written as $f=f_{D}+f_{J}$ with $f_{D} \in D(X, K)$ and $f_{J} \in J(I(X, K))$.

We denote by $\operatorname{Aut}(I(X, K))$ and $\operatorname{LAut}(I(X, K))$ the groups of usual and Lie automorphisms of $I(X, K)$, respectively. We will also deal with the group of bijective $K$-linear maps of $I(X, K)$, denoted by $\mathrm{GL}(I(X, K))$, and with the group $\operatorname{Inn}_{1}(I(X, K))$ of the inner automorphisms of $I(X, K)$ consisting of the conjugations by $\beta \in I(X, K)$ with $\beta_{D}=\delta$. 


\section{The LOWER Central Series of $J(I(X, K))$}

From now on $K$ will be a field and $X$ a finite connected poset of cardinality $n$.

Lemma 2.1. An element $f \in I(X, K)$ commutes with $e_{u v}$ if and only if $f(x, u)=0$ for $x<u, f(v, y)=0$ for $y>v$ and $f(u, u)=f(v, v)$.

Proof. We have

$$
\begin{aligned}
f e_{u v}=e_{u v} f & \Leftrightarrow \sum_{x \leq y} f(x, y) e_{x y} e_{u v}=\sum_{x \leq y} f(x, y) e_{u v} e_{x y} \\
& \Leftrightarrow \sum_{x \leq u \leq v} f(x, u) e_{x v}=\sum_{u \leq v \leq y} f(v, y) e_{u y},
\end{aligned}
$$

whence the desired property of $f$ follows.

We recall that the derived ideal of a $K$-Lie algebra $L$ is

$$
[L, L]=\operatorname{Span}_{K}\{[a, b]: a, b \in L\} .
$$

The sequence of Lie ideals

$$
L \supseteq[L, L] \supseteq[L,[L, L]] \supseteq \cdots
$$

is called the lower central series of $L$. It is clear that each term of this series is invariant under any automorphism of $L$.

Let $J_{1}$ be the derived ideal of $I(X, K)$ and

$$
J_{m}=\left[J_{1}, J_{m-1}\right]=\operatorname{Span}_{K}\left\{[f, g]: f \in J_{1}, g \in J_{m-1}\right\}, m \geq 2 .
$$

We thus have the following.

Proposition 2.2. If $\varphi \in \operatorname{LAut}(I(X, K))$, then $\varphi\left(J_{m}\right)=J_{m}$ for all $m \geq 1$.

Proposition 2.3. The ideal $J_{1}$ coincides with $J(I(X, K))$.

Proof. Let $f, g \in I(X, K)$. For all $x \in X$, we have

$$
(f g-g f)(x, x)=f(x, x) g(x, x)-g(x, x) f(x, x)=0 .
$$

Thus $[f, g] \in J(I(X, K))$.

On the other hand, if $x<y$ in $X$, then $e_{x y}=e_{x} e_{x y}-e_{x y} e_{x}=\left[e_{x}, e_{x y}\right] \in J_{1}$.

Proposition 2.4. Let $m$ be a positive integer. Then

$$
J_{m}=\operatorname{Span}_{K}\left\{e_{x y}: l(\lfloor x, y\rfloor) \geq m\right\}=J(I(X, K))^{m} .
$$

In particular, $J_{n}=J(I(X, K))^{n}=\{0\}$. It follows that $J_{m}$ is an ideal (and, therefore, a Lie ideal) of $I(X, K)$.

Proof. The statement is obvious for $m=1$ by Proposition 2.3 so let $m>1$.

Let $e_{u_{1} v_{1}}, \ldots, e_{u_{m} v_{m}} \in J(I(X, K)), i=1, \ldots, m$. If $e_{u_{1} v_{1}} \cdots e_{u_{m} v_{m}} \neq 0$, then $v_{i}=u_{i+1}, i=1, \ldots, m-1$. In this case, $u_{1}<u_{2}<\cdots<u_{m}<v_{m}$ and $e_{u_{1} v_{1}} \cdots e_{u_{m} v_{m}}=e_{u_{1} v_{m}}$ with $l\left(\left\lfloor u_{1}, v_{m}\right\rfloor\right) \geq m$. Therefore,

$$
J(I(X, K))^{m} \subseteq \operatorname{Span}_{K}\left\{e_{x y}: l(\lfloor x, y\rfloor) \geq m\right\} .
$$

Assume that $\operatorname{Span}_{K}\left\{e_{x y}: l(\lfloor x, y\rfloor) \geq k\right\} \subseteq J_{k}$ for some positive integer $k$ and let $x<y$ such that $l(\lfloor x, y\rfloor) \geq k+1$. We will show that $e_{x y} \in J_{k+1}$. Let $x=t_{1}<$ $\cdots<t_{k+2}=y$ be a chain in $\lfloor x, y\rfloor$ of length $k+1$. Then

$$
e_{x y}=e_{x t_{k+1}} e_{t_{k+1} y}-e_{t_{k+1} y} e_{x t_{k+1}}=\left[e_{x t_{k+1}}, e_{t_{k+1} y}\right] \text {. }
$$


Observe that $e_{t_{k+1} y} \in J(I(X, K))$ and $l\left(\left\lfloor x, t_{k+1}\right\rfloor\right) \geq k$. By the induction hypothesis, $e_{x t_{k+1}} \in J_{k}$ and, therefore, $e_{x y} \in J_{k+1}$, as we wanted. Thus,

$$
\operatorname{Span}_{K}\left\{e_{x y}: l(\lfloor x, y\rfloor) \geq m\right\} \subseteq J_{m} .
$$

Finally, let $J_{k} \subseteq J(I(X, K))^{k}$ for some positive integer $k$. If $f \in J(I(X, K))$ and $g \in J_{k}$, then $[f, g] \in J(I(X, K))^{k+1}$. Thus, $J_{m} \subseteq J(I(X, K))^{m}$.

Proposition 2.4 implies that the lower central series of $(J(I(X, K)),[]$,$) is$

$$
J(I(X, K)) \supseteq J(I(X, K))^{2} \supseteq \cdots \supseteq J(I(X, K))^{n-1} \supseteq J(I(X, K))^{n}=\{0\} .
$$

Proposition 2.5. The center $Z$ of $J(I(X, K))$ is

$$
Z=\operatorname{Span}_{K}\left\{e_{x y}: x<y, x \text { is minimal and } y \text { is maximal }\right\} .
$$

Proof. Let $u, v, x, y \in X$ such that $u<v, x<y, x$ is minimal and $y$ is maximal. Then $\left[e_{x y}, e_{u v}\right]=\delta_{y u} e_{x v}-\delta_{v x} e_{u y}$. If $y=u$, then $y<v$, which contradicts the maximality of $y$. If $v=x$, then $u<x$, which contradicts the minimality of $x$. Thus, $\left[e_{x y}, e_{u v}\right]=0$ and, therefore, $e_{x y} \in Z$.

Conversely, let $g=\sum_{x<y} g(x, y) e_{x y} \in Z$. Assume that $x$ is not minimal. Then there exists $u<x$. Using $g e_{u x}=e_{u x} g$ we conclude that $g(x, y)=0$ for all $y>x$ by Lemma 2.1. Similarly, if $y$ is not maximal, $g(x, y)=0$ for all $x<y$.

\section{Associative And Lie ideals of $I(X, K)$}

We begin this section by presenting some properties of associative and Lie ideals of $I(X, K)$ contained in $J(I(X, K))$.

Lemma 3.1. Let $I$ be a Lie ideal of $I(X, K)$ such that $I \subseteq J(I(X, K))$. If $f \in I$ and $f(x, y) \neq 0$, then $e_{x y} \in I$.

Proof. Let $f \in I$ such that $f(x, y) \neq 0$. Then $x<y$ since $I \subseteq J(I(X, K))$. Therefore, $f(x, y) e_{x y}=\left[\left[e_{x}, f\right], e_{y}\right] \in I$, whence $e_{x y} \in I$.

Corollary 3.2. Let $I$ be a Lie ideal of $I(X, K)$ such that $I \subseteq J(I(X, K))$. Then $\left.I=\operatorname{Span}_{K}\left\{e_{x y}: e_{x y} \in I\right\} 1\right]$

Given a subset $S$ of $I(X, K)$, we will denote by $\langle S\rangle$ (resp. $\left.\langle S\rangle_{L}\right)$ the ideal (resp. Lie ideal) of $I(X, K)$ generated by $S$.

Lemma 3.3. For all $x \leq y$ one has $\left\langle e_{x y}\right\rangle=\operatorname{Span}_{K}\left\{e_{u v}: u \leq x \leq y \leq v\right\}$.

Proof. If $u \leq x \leq y \leq v$, then $e_{u v}=e_{u x} e_{x y} e_{y v}$. Conversely, the product $e_{a b} e_{x y} e_{c d}$ is nonzero if and only if $b=x$ and $y=c$, in which case it equals $e_{a d}$ with $a \leq x \leq$ $y \leq d$.

Lemma 3.4. Let $e_{x y} \in\langle S\rangle$, where $S \subseteq J(I(X, K))$. Then there are $f \in S$ and $x \leq u<v \leq y$ such that $f(u, v) \neq 0$.

Proof. Write $e_{x y}=\sum_{i} g_{i} f_{i} h_{i}$ with $g_{i}, h_{i} \in I(X, K)$ and $f_{i} \in S$. If $f_{i}(u, v)=0$ for all $x \leq u<v \leq y$, then $1=e_{x y}(x, y)=\sum_{i} \sum_{x \leq u<v \leq y} g_{i}(x, u) f_{i}(u, v) h_{i}(v, y)=0$, a contradiction.

Corollary 3.5. If $S \subseteq J(I(X, K))$, then $\langle S\rangle=\langle S\rangle_{L}$. Therefore, every Lie ideal of $I(X, K)$ contained in $J(I(X, K))$ is an ideal.

\footnotetext{
${ }^{1}$ For an associative ideal this is well-known (see [8, Lemma 3.1]).
} 
Proof. Clearly, $\langle S\rangle_{L} \subseteq\langle S\rangle$. To prove that $\langle S\rangle \subseteq\langle S\rangle_{L}$, we only need to show by Corollary 3.2 that $e_{x y} \in\langle S\rangle_{L}$ whenever $e_{x y} \in\langle S\rangle$. Let $e_{x y} \in\langle S\rangle$. By Lemma 3.4 there are $f \in S$ and $x \leq u<v \leq y$ such that $f(u, v) \neq 0$. Then $e_{x y}=f(u, v)^{-1}\left[\left[e_{x u}, f\right], e_{v y}\right] \in\langle S\rangle_{L}$.

Proposition 3.6. Let $I \subseteq J_{m}$ be an ideal of $I(X, K)$ such that $\operatorname{dim} \frac{I}{I \cap J_{m+1}}=1$. Then there exists a unique $e_{x y} \in J_{m}-J_{m+1}$ such that any $f \in I-J_{m+1}$ is of the form $f=f(x, y) e_{x y}+j_{x y}$, where $j_{x y} \in J_{m+1}$.

Proof. Let $f \in I-J_{m+1}$. Then there is $e_{x y} \in J_{m}-J_{m+1}$ such that $f(x, y) \neq 0$. By Lemma 3.1, $e_{x y} \in I$. Since $\operatorname{dim} \frac{I}{I \cap J_{m+1}}=1, e_{x y}$ is the only $e_{u v} \in J_{m}-J_{m+1}$ that belongs to $I$. Thus, $f-f(x, y) e_{x y} \in J_{m+1}$.

Lemma 3.7. Let $x \leq y$ and $u \leq v$. If $y \not \leq u$, then $\left\langle e_{x y}\right\rangle\left\langle e_{u v}\right\rangle=\{0\}$.

Proof. Let $f \in\left\langle e_{x y}\right\rangle$ and $g \in\left\langle e_{u v}\right\rangle$. If there are $a \leq b$ such that

$$
0 \neq(f g)(a, b)=\sum_{a \leq c \leq b} f(a, c) g(c, b),
$$

then $f(a, c) \neq 0$ and $g(c, b) \neq 0$ for some $a \leq c \leq b$. It follows by Lemma 3.3 that $a \leq x \leq y \leq c$ and $c \leq u \leq v \leq b$, whence $y \leq u$, a contradiction.

\section{The Decomposition of LAut $(I(X, K))$ into A Semidirect product}

Let $L_{i}=\operatorname{Span}_{K}\left\{e_{x y}: e_{x y} \in J_{i}-J_{i+1}\right\}=\operatorname{Span}_{K}\left\{e_{x y}: l(\lfloor x, y\rfloor)=i\right\}$, for any integer $i \geq 0$, where $J_{0}:=I(X, K)$. Then $J_{i}=\bigoplus_{k \geq i} L_{k}$ as $K$-vector spaces, $i \geq 0$.

Definition 4.1. Given $\varphi \in \operatorname{LAut}(I(X, K))$, set $\widetilde{\varphi}: I(X, K) \rightarrow I(X, K)$ to be the $K$-linear map defined as follows: for any $e_{x y}$, if $e_{x y} \in L_{i}$, then

$$
\varphi\left(e_{x y}\right)=\widetilde{\varphi}\left(e_{x y}\right)+j_{x y},
$$

where $\widetilde{\varphi}\left(e_{x y}\right) \in L_{i}$ and $j_{x y} \in J_{i+1}$.

Clearly, $\widetilde{\varphi}$ is well-defined in view of Proposition 2.2 .

Proposition 4.2. The map $\varphi \mapsto \widetilde{\varphi}$ is a group homomorphism from $\operatorname{LAut}(I(X, K))$ to $\operatorname{GL}(I(X, K))$.

Proof. We first prove that $\widetilde{\varphi} \in \operatorname{GL}(I(X, K))$. Since $\widetilde{\varphi}$ is linear, it suffices to check that $\widetilde{\varphi}$ is onto. Let $x \leq y$ and take $g \in I(X, K)$ such that $\varphi(g)=e_{x y}$. If $e_{x y} \in L_{i}$, then, by Proposition [2.2, $g \in J_{i}$, so $g=f+h$ with $f \in L_{i}$ and $h \in J_{i+1}$. It follows that $e_{x y}=\varphi(g)=\varphi(f)+\varphi(h)=\widetilde{\varphi}(f)+j+\varphi(h)$, where $j \in J_{i+1}$. Thus, $e_{x y}-\widetilde{\varphi}(f)=j+\varphi(h)$ in which $e_{x y}-\widetilde{\varphi}(f) \in L_{i}$ and $j+\varphi(h) \in J_{i+1}$. Therefore, $\widetilde{\varphi}(f)=e_{x y}$ (and $j+\varphi(h)=0$ ), which implies that $\widetilde{\varphi}$ is onto.

Let $\varphi, \psi \in \operatorname{LAut}(I(X, K))$. We need to prove that $\widetilde{\varphi \circ \psi}=\widetilde{\varphi} \circ \widetilde{\psi}$. Let $x \leq y$ with $e_{x y} \in L_{i}$. Then $\psi\left(e_{x y}\right)=\widetilde{\psi}\left(e_{x y}\right)+j_{x y}$, where $j_{x y} \in J_{i+1}$, so $\varphi\left(\psi\left(e_{x y}\right)\right)=$ $\varphi\left(\widetilde{\psi}\left(e_{x y}\right)\right)+\varphi\left(j_{x y}\right)=\widetilde{\varphi}\left(\widetilde{\psi}\left(e_{x y}\right)\right)+j_{x y}^{\prime}+\varphi\left(j_{x y}\right)$ for some $j_{x y}^{\prime} \in J_{i+1}$. Since $\widetilde{\varphi}\left(\widetilde{\psi}\left(e_{x y}\right)\right) \in$ $L_{i}$ and $j_{x y}^{\prime}+\varphi\left(j_{x y}\right) \in J_{i+1}$, then $(\widetilde{\varphi \circ \psi})\left(e_{x y}\right)=\widetilde{\varphi}\left(\widetilde{\psi}\left(e_{x y}\right)\right)$.

Let us introduce $B_{i}=B \cap J_{i}$ and $X_{<}^{2}=\left\{(x, y) \in X^{2}: x<y\right\}$. 
Lemma 4.3. Let $\varphi \in \operatorname{LAut}(I(X, K))$. There exist a bijection $\theta=\theta_{\varphi}: B \rightarrow B$ with $\theta\left(B_{i}\right) \subseteq B_{i}$ and a map $\sigma=\sigma_{\varphi}: X_{<}^{2} \rightarrow K^{*}$ such that, for any $e_{x y} \in B$,

$$
\widetilde{\varphi}\left(e_{x y}\right)=\sigma(x, y) \theta\left(e_{x y}\right) .
$$

Proof. Suppose that $e_{x y} \in B_{i}-B_{i+1}$. We will introduce $I_{x y}=\left\langle e_{x y}\right\rangle /\left(\left\langle e_{x y}\right\rangle \cap J_{i+1}\right)$. Observe that $\operatorname{dim}\left(I_{x y}\right)=1$ since any $e_{u v} \in\left\langle e_{x y}\right\rangle$ with $e_{u v} \neq e_{x y}$ belongs to $J_{i+1}$ by Lemma 3.3

Note that $\varphi\left(\left\langle e_{x y}\right\rangle\right) \subseteq J_{i}$ by Proposition 2.2 and $\varphi\left(\left\langle e_{x y}\right\rangle\right)$ is an ideal of $I(X, K)$ by Corollary 3.5. Moreover, $\varphi\left(\left\langle e_{x y}\right\rangle\right) /\left(\varphi\left(\left\langle e_{x y}\right\rangle\right) \cap J_{i+1}\right)$ and $I_{x y}$ are isomorphic as Lie algebras. Hence $\operatorname{dim}\left(\varphi\left(\left\langle e_{x y}\right\rangle\right) /\left(\varphi\left(\left\langle e_{x y}\right\rangle\right) \cap J_{i+1}\right)\right)=\operatorname{dim}\left(I_{x y}\right)=1$. By Proposition 3.6 there are $e_{u v} \in B_{i}-B_{i+1}, k \in K^{*}$ and $g \in J_{i+1}$ such that $\varphi\left(e_{x y}\right)=k e_{u v}+g$. Hence, $\widetilde{\varphi}\left(e_{x y}\right)=k e_{u v}$. We thus define $\theta: B \rightarrow B$ by $\theta\left(e_{x y}\right)=e_{u v}$ and $\sigma: X_{<}^{2} \rightarrow K^{*}$ by $\sigma(x, y)=k$. Clearly, $\theta\left(B_{i}\right) \subseteq B_{i}$.

It remains to prove that $\theta$ is a bijection. Since $B$ is finite, it suffices to establish the injectivity of $\theta$. Suppose that $\widetilde{\varphi}\left(e_{x y}\right)=\sigma(x, y) e_{u v}$ and $\widetilde{\varphi}\left(e_{a b}\right)=\sigma(a, b) e_{u v}$ for some $e_{x y}, e_{a b} \in B_{i}-B_{i+1}$. Then $\varphi\left(e_{x y}\right)=\sigma(x, y) e_{u v}+g$ and $\varphi\left(e_{a b}\right)=\sigma(a, b) e_{u v}+h$ for some $g, h \in J_{i+1}$. Consequently, $\varphi\left(\sigma(a, b) e_{x y}-\sigma(x, y) e_{a b}\right)=\sigma(a, b) g-\sigma(x, y) h \in$ $J_{i+1}$. It follows that $\sigma(a, b) e_{x y}-\sigma(x, y) e_{a b} \in J_{i+1}$ by Proposition 2.2. Since $\sigma(x, y), \sigma(a, b) \in K^{*}$, then $e_{x y}=e_{a b}($ and $\sigma(x, y)=\sigma(a, b))$.

Lemma 4.4. Let $\varphi \in \operatorname{LAut}(I(X, K))$ and $e_{x y} \in L_{i}$, where $i>0$. Then $\varphi\left(e_{x y}\right)-$ $\widetilde{\varphi}\left(e_{x y}\right) \in\left\langle\theta_{\varphi}\left(e_{x y}\right)\right\rangle \cap J_{i+1}$.

Proof. Write $\varphi\left(e_{x y}\right)=\widetilde{\varphi}\left(e_{x y}\right)+g$ with $g \in J_{i+1}$. Since $e_{x y}=\left[e_{x}, e_{x y}\right]$, then

$$
\widetilde{\varphi}\left(e_{x y}\right)+g=\varphi\left(\left[e_{x}, e_{x y}\right]\right)=\left[\varphi\left(e_{x}\right), \varphi\left(e_{x y}\right)\right]=\left[\varphi\left(e_{x}\right), \widetilde{\varphi}\left(e_{x y}\right)\right]+\left[\varphi\left(e_{x}\right), g\right] .
$$

But $\widetilde{\varphi}\left(e_{x y}\right) \in\left\langle\theta_{\varphi}\left(e_{x y}\right)\right\rangle$ by Lemma 4.3, so

$$
g-\left[\varphi\left(e_{x}\right), g\right]=\left[\varphi\left(e_{x}\right), \widetilde{\varphi}\left(e_{x y}\right)\right]-\widetilde{\varphi}\left(e_{x y}\right) \in\left\langle\theta_{\varphi}\left(e_{x y}\right)\right\rangle .
$$

Similarly $e_{x y}=\left[e_{x y}, e_{y}\right]$ implies that

$$
g-\left[g, \varphi\left(e_{y}\right)\right] \in\left\langle\theta_{\varphi}\left(e_{x y}\right)\right\rangle .
$$

Now, using (2) and (3) we have

$$
g-\left[\left[\varphi\left(e_{x}\right), g\right], \varphi\left(e_{y}\right)\right]=g-\left[g, \varphi\left(e_{y}\right)\right]+\left[g-\left[\varphi\left(e_{x}\right), g\right], \varphi\left(e_{y}\right)\right] \in\left\langle\theta_{\varphi}\left(e_{x y}\right)\right\rangle .
$$

Choose $h \in J_{i+1}$, such that $g=\varphi(h)$. Then

$$
\left[\left[\varphi\left(e_{x}\right), g\right], \varphi\left(e_{y}\right)\right]=\left[\left[\varphi\left(e_{x}\right), \varphi(h)\right], \varphi\left(e_{y}\right)\right]=\varphi\left(\left[\left[e_{x}, h\right], e_{y}\right]\right)=\varphi\left(h(x, y) e_{x y}\right) .
$$

But $h(x, y)=0$ by Proposition 2.4, so $\left[\left[\varphi\left(e_{x}\right), g\right], \varphi\left(e_{y}\right)\right]=0$ and the result follows from (4).

Proposition 4.5. Let $\varphi \in \operatorname{LAut}(I(X, K))$. Then $\widetilde{\varphi} \in \operatorname{LAut}(I(X, K))$.

Proof. Since $\widetilde{\varphi} \in \mathrm{GL}(I(X, K))$ by Proposition 4.2 , it remains to prove $\widetilde{\varphi}\left(\left[e_{x y}, e_{u v}\right]\right)=$ $\left[\widetilde{\varphi}\left(e_{x y}\right), \widetilde{\varphi}\left(e_{u v}\right)\right]$, for all $x \leq y$ and $u \leq v$.

The case $e_{x y}, e_{u v} \in L_{0}=D(X, K)$ is trivial because $\left[L_{0}, L_{0}\right]=\{0\}$. If $e_{x y} \in L_{0}$ and $e_{u v} \in L_{i}$ with $i>0$, then $\varphi\left(\left[e_{x y}, e_{u v}\right]\right)=\left[\varphi\left(e_{x y}\right), \varphi\left(e_{u v}\right)\right]=\left[\widetilde{\varphi}\left(e_{x y}\right), \widetilde{\varphi}\left(e_{u v}\right)\right]+j$, where $j \in J_{i+1}$. Since $\left[L_{0}, L_{i}\right] \subseteq L_{i}$, then $\widetilde{\varphi}\left(\left[e_{x y}, e_{u v}\right]\right)=\left[\widetilde{\varphi}\left(e_{x y}\right), \widetilde{\varphi}\left(e_{u v}\right)\right]$.

Let $e_{x y} \in L_{i}$ and $e_{u v} \in L_{j}$ with $i, j>0$. By Lemmas 4.3 and 4.4, $\varphi\left(e_{x y}\right)=$ $\alpha e_{a b}+g$ and $\varphi\left(e_{u v}\right)=\beta e_{c d}+h$, where $\alpha, \beta \in K^{*}, e_{a b} \in L_{i}, e_{c d} \in L_{j}, g \in\left\langle e_{a b}\right\rangle \cap J_{i+1}$, $h \in\left\langle e_{c d}\right\rangle \cap J_{j+1}$. Thus, $\widetilde{\varphi}\left(e_{x y}\right)=\alpha e_{a b}$ and $\widetilde{\varphi}\left(e_{u v}\right)=\beta e_{c d}$. If $\left[e_{x y}, e_{u v}\right]=0$, then $\left[\widetilde{\varphi}\left(e_{x y}\right), \widetilde{\varphi}\left(e_{u v}\right)\right]=0$. Indeed, assume that $e_{a b} e_{c d} \neq 0$, i.e., $b=c$. Then 
$0=\left[\varphi\left(e_{x y}\right), \varphi\left(e_{u v}\right)\right]=\alpha \beta e_{a d}+p$, where $p=\alpha\left[e_{a b}, h\right]+\beta\left[g, e_{c d}\right]+[g, h]$. Observe from Lemma 3.3 that $p \in\left\langle e_{a d}\right\rangle \cap J_{k+1}$, where $k=l(\lfloor a, d\rfloor)$, a contradiction. The case $e_{c d} e_{a b} \neq 0$ is similar. Let now $\left[e_{x y}, e_{u v}\right] \neq 0$. Then applying the above argument to $e_{a b}, e_{c d}$ and $\varphi^{-1}$ we conclude that $\left[e_{a b}, e_{c d}\right] \neq 0$, because $\alpha^{-1} e_{x y}=\widetilde{\varphi^{-1}}\left(e_{a b}\right)$ and $\beta^{-1} e_{u v}=\widetilde{\varphi^{-1}}\left(e_{c d}\right)$. Let $b=c$ and write $\varphi\left(\left[e_{x y}, e_{u v}\right]\right)=\left[\varphi\left(e_{x y}\right), \varphi\left(e_{u v}\right)\right]=$ $\alpha \beta e_{a d}+p$ as above. If $l(\lfloor a, d\rfloor)=k$, then $p \in J_{k+1}$, whence $\widetilde{\varphi}\left(\left[e_{x y}, e_{u v}\right]\right)=\alpha \beta e_{a d}=$ $\left[\widetilde{\varphi}\left(e_{x y}\right), \widetilde{\varphi}\left(e_{u v}\right)\right]$. If $a=d$, the proof is analogous.

Corollary 4.6. The map $\varphi \mapsto \widetilde{\varphi}$ is an endomorphism of the group $\operatorname{LAut}(I(X, K))$.

Definition 4.7. Denote by $\widetilde{\operatorname{LAut}}(I(X, K))$ the image of $\varphi \mapsto \widetilde{\varphi}$. A Lie automorphism from $\widetilde{\operatorname{LAut}}(I(X, K))$ will be called elementary.

Remark 4.8. Observe that $\varphi$ is elementary if and only if $\varphi\left(L_{i}\right) \subseteq L_{i}$, for all $i \geq 0$.

We will also introduce the temporary notation $N$ for the kernel of $\varphi \mapsto \widetilde{\varphi}$. We will soon see that $N=\operatorname{Inn}_{1}(I(X, K))$.

Remark 4.9. For any $\varphi \in \operatorname{LAut}(I(X, K))$ one has $\widetilde{\widetilde{\varphi}}=\widetilde{\varphi}$. In particular, $\varphi \circ(\widetilde{\varphi})^{-1}$ belongs to $N$.

Lemma 4.10. Let $\varphi \in N$. Then $\varphi\left(e_{x}\right) \in\left\langle e_{x}\right\rangle$ for all $x \in X$.

Proof. Take $u<v$ such that $x \notin\lfloor u, v\rfloor$. Write $\varphi\left(e_{x}\right)=e_{x}+j_{x}$ and $\varphi\left(e_{u}\right)=e_{u}+j_{u}$, where $j_{x}, j_{u} \in J(I(X, K))$. We need to show that $j_{x}(u, v)=0$. Applying $\varphi$ to $\left[e_{x}, e_{u}\right]=0$, we obtain

$$
\left[e_{x}, j_{u}\right]+\left[j_{x}, e_{u}\right]+\left[j_{x}, j_{u}\right]=0 .
$$

Calculating the value of this sum at $(u, v)$, we have

$$
j_{x}(u, v)=\left[j_{x}, j_{u}\right](u, v)=\sum_{u<w<v}\left(j_{x}(u, w) j_{u}(w, v)-j_{u}(u, w) j_{x}(w, v)\right) .
$$

We will prove that $j_{x}(u, v)=0$ by induction on $l(\lfloor u, v\rfloor)$. If $l(\lfloor u, v\rfloor)=1$, then $j_{x}(u, v)=0$, since there is no $w$ with $u<w<v$ on the right-hand side of (5). If $l(\lfloor u, v\rfloor)>1$, then $l(\lfloor u, w\rfloor)$ and $l(\lfloor w, v\rfloor)$ are strictly less than $l(\lfloor u, v\rfloor)$ for any $u<w<v$. Since moreover $x \notin\lfloor u, w\rfloor$ and $x \notin\lfloor w, v\rfloor$, by induction hypothesis $j_{x}(u, w)=j_{x}(w, v)=0$, whence $j_{x}(u, v)=0$ by (5) .

Corollary 4.11. Let $\varphi \in N$. Then $\varphi\left(e_{x}\right) \varphi\left(e_{y}\right)=0$ for all $x \neq y$.

Proof. Notice that $\varphi\left(e_{x}\right)$ commutes with $\varphi\left(e_{y}\right)$, since $\left[\varphi\left(e_{x}\right), \varphi\left(e_{y}\right)\right]=\varphi\left(\left[e_{x}, e_{y}\right]\right)=$ 0 . If $x \not \leq y$, then the result follows from Lemmas 3.7 and 4.10 . If $x<y$, then $y \not \leq x$, so $\varphi\left(e_{y}\right) \varphi\left(e_{x}\right)=0$ by the previous case, whence $\varphi\left(e_{x}\right) \varphi\left(e_{y}\right)=0$.

Lemma 4.12. Let $\varphi \in N$. Then $\varphi(\delta)=\delta$.

Proof. Since $\delta$ is central, then $\varphi(\delta)$ is also central, in particular, it is diagonal, by [16. Corollary 1.3.15]. On the other hand, $\delta=\sum_{x \in X} e_{x}$, whence $\varphi(\delta)=\varphi(\delta)_{D}=$ $\sum_{x \in X} \varphi\left(e_{x}\right)_{D}=\sum_{x \in X} e_{x}=\delta$.

Corollary 4.13. Let $\varphi \in N$. Then $\varphi\left(e_{x}\right)$ is an idempotent for all $x \in X$.

Proof. By Lemma 4.12 we have $\delta=\varphi(\delta)=\sum_{x \in X} \varphi\left(e_{x}\right)$. Multiplying this by $\varphi\left(e_{x}\right)$ and using Corollary 4.11 we obtain $\varphi\left(e_{x}\right)^{2}=\varphi\left(e_{x}\right)$. 
Proposition 4.14. The group $N$ coincides with $\operatorname{Inn}_{1}(I(X, K))$.

Proof. If $\beta=\delta+\rho_{1}$ with $\rho_{1} \in J_{1}$ then $\beta^{-1}=\delta+\rho_{2}$ with $\rho_{2} \in J_{1}$. For any $e_{x y} \in L_{i}$ we have $\beta e_{x y} \beta^{-1}=\left(\delta+\rho_{1}\right) e_{x y}\left(\delta+\rho_{2}\right)=e_{x y}+\rho$, where $\rho \in J_{i+1}$. Hence, $\operatorname{Inn}_{1}(I(X, K)) \subseteq N$. We will prove the converse inclusion.

Let $\varphi \in N$. Define $\beta=\sum_{x \in X} \varphi\left(e_{x}\right) e_{x}$. Since $\widetilde{\varphi}=$ id, we have $\varphi\left(e_{x}\right)_{D}=e_{x}$, whence $\beta_{D}=\sum_{x \in X} e_{x}=\delta$. Clearly, $\beta e_{x}=\varphi\left(e_{x}\right) e_{x}$. In view of Corollaries 4.11 and 4.13 we similarly obtain $\varphi\left(e_{x}\right) \beta=\varphi\left(e_{x}\right) e_{x}$. Thus, $\varphi\left(e_{x}\right)=\beta e_{x} \beta^{-1}$.

Denote by $\xi_{\beta}$ the conjugation by $\beta$. Then $\xi_{\beta} \in \operatorname{Inn}_{1}(I(X, K)) \subseteq N$. Set $\psi:=$ $\left(\xi_{\beta}\right)^{-1} \circ \varphi$. Then $\psi \in N$ and moreover, $\psi\left(e_{x}\right)=e_{x}$ for all $x \in X$. Applying $\psi$ to $e_{x y}=\left[e_{x},\left[e_{x y}, e_{y}\right]\right] \in L_{i}$ with $i>0$, we have

$$
\psi\left(e_{x y}\right)=\left[e_{x},\left[\psi\left(e_{x y}\right), e_{y}\right]\right]=\left[e_{x},\left[e_{x y}+j_{x y}, e_{y}\right]\right]=\left(1+j_{x y}(x, y)\right) e_{x y}=e_{x y},
$$

as $j_{x y} \in J_{i+1}$. Thus, $\psi=$ id and so $\varphi=\xi_{\beta}$.

As an immediate consequence of Remark 4.9 and Proposition 4.14 we have the following.

Theorem 4.15. The group $\operatorname{LAut}(I(X, K))$ is isomorphic to the semidirect product $\operatorname{Inn}_{1}(I(X, K)) \rtimes \widetilde{\operatorname{LAut}}(I(X, K))$.

\section{Elementary Lie automorphisms and admissible pairs}

Let $\varphi \in \widetilde{\operatorname{LAut}}(I(X, K))$ and consider $\theta=\theta_{\varphi}$ and $\sigma=\sigma_{\varphi}$ as in Lemma4.3 Then, for any $e_{x y} \in B$,

$$
\varphi\left(e_{x y}\right)=\sigma(x, y) \theta\left(e_{x y}\right) .
$$

Remark 5.1. Let $\varphi \in \widetilde{\operatorname{LAut}}(I(X, K))$. Then $\theta_{\varphi^{-1}}=\left(\theta_{\varphi}\right)^{-1}$ and $\sigma_{\varphi^{-1}}(u, v)=$ $\sigma_{\varphi}(x, y)^{-1}$, where $\theta_{\varphi}\left(e_{x y}\right)=e_{u v}$.

Lemma 5.2. Let $\varphi \in \widetilde{\operatorname{LAut}}(I(X, K)), \theta=\theta_{\varphi}, \sigma=\sigma_{\varphi}$ and $x<y<z$. Then either $\theta\left(e_{x z}\right)=\theta\left(e_{x y}\right) \theta\left(e_{y z}\right)$ in which case $\sigma(x, z)=\sigma(x, y) \sigma(y, z)$, or $\theta\left(e_{x z}\right)=\theta\left(e_{y z}\right) \theta\left(e_{x y}\right)$ in which case $\sigma(x, z)=-\sigma(x, y) \sigma(y, z)$.

Proof. By (6) we have

$$
\sigma(x, z) \theta\left(e_{x z}\right)=\varphi\left(e_{x z}\right)=\left[\varphi\left(e_{x y}\right), \varphi\left(e_{y z}\right)\right]=\sigma(x, y) \sigma(y, z)\left[\theta\left(e_{x y}\right), \theta\left(e_{y z}\right)\right] .
$$

Since $\left[\theta\left(e_{x y}\right), \theta\left(e_{y z}\right)\right] \neq 0$ and $\theta\left(e_{x y}\right), \theta\left(e_{y z}\right) \in B$, then either $\theta\left(e_{x y}\right) \theta\left(e_{y z}\right) \neq 0$, in which case $\left[\theta\left(e_{x y}\right), \theta\left(e_{y z}\right)\right]=\theta\left(e_{x y}\right) \theta\left(e_{y z}\right)$, or $\theta\left(e_{y z}\right) \theta\left(e_{x y}\right) \neq 0$, in which case $\left[\theta\left(e_{x y}\right), \theta\left(e_{y z}\right)\right]=-\theta\left(e_{y z}\right) \theta\left(e_{x y}\right)$. Thus, the result follows from (77).

Definition 5.3. Let $\theta: B \rightarrow B$ be a bijection and $\sigma: X_{<}^{2} \rightarrow K^{*}$ a map. We say that $\sigma$ is compatible with $\theta$ if $\sigma(x, z)=\sigma(x, y) \sigma(y, z)$ whenever $\theta\left(e_{x z}\right)=$ $\theta\left(e_{x y}\right) \theta\left(e_{y z}\right)$, and $\sigma(x, z)=-\sigma(x, y) \sigma(y, z)$ whenever $\theta\left(e_{x z}\right)=\theta\left(e_{y z}\right) \theta\left(e_{x y}\right)$.

The above definition makes sense, as $e_{x y}, e_{u v} \in B$ commute if and only if $e_{x y} e_{u v}=e_{u v} e_{x y}=0$.

Lemma 5.4. Let $\varphi \in \widetilde{\operatorname{LAut}}(I(X, K))$ and $\theta=\theta_{\varphi}$. For any maximal chain $C$ : $u_{1}<u_{2}<\cdots<u_{m}$ in $X$ there exists a maximal chain $D: v_{1}<v_{2}<\cdots<v_{m}$ in $X$ such that one of the following two conditions holds:

(i) $\theta\left(e_{u_{i} u_{j}}\right)=e_{v_{i} v_{j}}$ for all $1 \leq i<j \leq m$; 
(ii) $\theta\left(e_{u_{i} u_{j}}\right)=e_{v_{m-j+1} v_{m-i+1}}$ for all $1 \leq i<j \leq m$.

Proof. We first construct the chain $v_{1}<v_{2}<\cdots<v_{m}$ and then prove that it is maximal. If $m=2$, then $\theta\left(e_{u_{1} u_{2}}\right)=e_{v_{1} v_{2}}$ by (6). Let $m>2$ and consider $u_{1}<u_{2}<u_{3}$. By Lemma 5.2 we have two cases.

Case 1. There are $v_{1}<v_{2}<v_{3}$ such that $\theta\left(e_{u_{1} u_{2}}\right)=e_{v_{1} v_{2}}, \theta\left(e_{u_{2} u_{3}}\right)=e_{v_{2} v_{3}}$ and $\theta\left(e_{u_{1} u_{3}}\right)=e_{v_{1} v_{3}}$. If $m=3$, then we are done. If $m>3$, then there are $u<v$, such that $\theta\left(e_{u_{3} u_{4}}\right)=e_{u v}$. Applying Lemma 5.2 to $u_{2}<u_{3}<u_{4}$, we conclude that either $u=v_{3}$ or $v=v_{2}$. Now, applying the same lemma to $u_{1}<u_{3}<u_{4}$, we have either $u=v_{3}$ or $v=v_{1}$. Since $v_{1} \neq v_{2}$, then $u=v_{3}$. Hence, we may define $v_{4}:=v$, and we have $v_{1}<v_{2}<v_{3}<v_{4}$ and $\theta\left(e_{u_{3} u_{4}}\right)=e_{v_{3} v_{4}}, \theta\left(e_{u_{2} u_{4}}\right)=e_{v_{2} v_{4}}$, $\theta\left(e_{u_{1} u_{4}}\right)=e_{v_{1} v_{4}}$ by Lemma 5.2. Repeating the same argument, we consecutively construct $v_{1}<v_{2}<\cdots<v_{m}$ such that $\theta\left(e_{u_{i} u_{j}}\right)=e_{v_{i} v_{j}}$ for all $1 \leq i<j \leq m$.

Case 2. There are $v_{m-2}<v_{m-1}<v_{m}$ such that $\theta\left(e_{u_{1} u_{2}}\right)=e_{v_{m-1} v_{m}}, \theta\left(e_{u_{2} u_{3}}\right)=$ $e_{v_{m-2} v_{m-1}}$ and $\theta\left(e_{u_{1} u_{3}}\right)=e_{v_{m-2} v_{m}}$. This case is analogous to Case 1. Repeating a similar argument, we consecutively construct $v_{1}<v_{2}<\cdots<v_{m}$ such that $\theta\left(e_{u_{i} u_{j}}\right)=e_{v_{m-j+1} v_{m-i+1}}$ for all $1 \leq i<j \leq m$.

Now we prove that the constructed chain $v_{1}<v_{2}<\cdots<v_{m}$ is maximal. Since $u_{1}<u_{2}<\cdots<u_{m}$ is maximal, then $l\left(\left\lfloor u_{i}, u_{i+1}\right\rfloor\right)=1$ for all $i=1, \ldots, m-1$. The latter is equivalent to $e_{u_{i} u_{i+1}} \in B_{1}-B_{2}$. If $\theta\left(e_{u_{i} u_{i+1}}\right)=e_{v_{i} v_{i+1}}$, then $e_{v_{i} v_{i+1}} \in$ $B_{1}-B_{2}$ and hence $l\left(\left\lfloor v_{i}, v_{i+1}\right\rfloor\right)=1$. If $\theta\left(e_{u_{i} u_{i+1}}\right)=e_{v_{m-i} v_{m-i+1}}$, then we similarly obtain $l\left(\left\lfloor v_{m-i}, v_{m-i+1}\right\rfloor\right)=1$ for all $i=1, \ldots, m-1$. Both cases imply that there cannot exist $v$ such that $v_{i}<v<v_{i+1}$ for some $i=1, \ldots, m-1$. There cannot also exist $v_{0}$ such that $v_{0}<v_{1}$ and $v_{m+1}$ such that $v_{m+1}>v_{m}$. Indeed, $u_{1}$ is minimal and $u_{m}$ is maximal in $X$, so that $e_{u_{1} u_{m}} \in Z$ by Proposition 2.5. Hence $e_{v_{1} v_{m}}=\sigma\left(u_{1}, u_{m}\right)^{-1} \varphi\left(e_{u_{1} u_{m}}\right) \in Z$. Thus, $v_{1}$ is minimal and $v_{m}$ is maximal.

Definition 5.5. Let $\theta: B \rightarrow B$ be a bijection and $C$ a maximal chain in $X$. We say that $\theta$ is increasing (resp. decreasing) on $C$ if Lemma 5.4(i) (resp. Lemma 5.4 (ii)) holds. Moreover, we say that $\theta$ is monotone on maximal chains in $X$ if, for any maximal chain $C, \theta$ is increasing or decreasing on $C$.

Remark 5.6. If a bijection $\theta: B \rightarrow B$ is monotone on maximal chains in $X$, then so is $\theta^{-1}$, since $X$ is finite.

Lemma 5.7. Let $\varphi \in \widetilde{\operatorname{LAut}}(I(X, K)), \theta=\theta_{\varphi}, x<y$ and $\theta\left(e_{x y}\right)=e_{u v}$. Then for all $z \in X$ :

$$
\varphi\left(e_{z}\right)(v, v)-\varphi\left(e_{z}\right)(u, u)= \begin{cases}-1, & z=x, \\ 1, & z=y, \\ 0, & z \notin\{x, y\} .\end{cases}
$$

Proof. Let $e_{x y} \in B_{m}-B_{m+1}$. By (6), we have $\varphi\left(e_{x y}\right)=\sigma(x, y) e_{u v}$, where $\sigma(x, y) \in$ $K^{*}$ and $e_{u v} \in B_{m}-B_{m+1}$. Then applying $\varphi$ to $\left[e_{x}, e_{x y}\right]=e_{x y}$ and using the fact that $\varphi\left(e_{x}\right) \in D(X, K)$, we obtain

$$
\sigma(x, y) e_{u v}=\sigma(x, y)\left[\varphi\left(e_{x}\right), e_{u v}\right]=\sigma(x, y)\left(\varphi\left(e_{x}\right)(u, u)-\varphi\left(e_{x}\right)(v, v)\right) e_{u v} .
$$

Since $\sigma(x, y) \neq 0$, we deduce from (9) that $\varphi\left(e_{x}\right)(u, u)-\varphi\left(e_{x}\right)(v, v)=1$.

Similarly one gets $\varphi\left(e_{y}\right)(u, u)-\varphi\left(e_{y}\right)(v, v)=-1$ from $\left[e_{y}, e_{x y}\right]=-e_{x y}$ and $\varphi\left(e_{z}\right)(u, u)-\varphi\left(e_{z}\right)(v, v)=0(z \notin\{x, y\})$ from $\left[e_{z}, e_{x y}\right]=0$. 
Lemma 5.8. Fix an arbitrary $u_{0} \in X$. Given $\varphi, \psi \in \widetilde{\operatorname{LAut}}(I(X, K))$, if $\theta_{\varphi}=\theta_{\psi}$ and $\varphi\left(e_{z}\right)\left(u_{0}, u_{0}\right)=\psi\left(e_{z}\right)\left(u_{0}, u_{0}\right)$ for all $z \in X$, then $\varphi\left(e_{z}\right)=\psi\left(e_{z}\right)$ for all $z \in X$.

Proof. Given $v \in X$, choose a walk $\Gamma: u_{0}, u_{1}, \ldots, u_{m}=v$ from $u_{0}$ to $v$. For each $0 \leq i \leq m-1$ let $v_{i}<w_{i}$ be such that

$$
\theta_{\varphi}\left(e_{v_{i} w_{i}}\right)= \begin{cases}e_{u_{i} u_{i+1}}, & u_{i}<u_{i+1} \\ e_{u_{i+1} u_{i}}, & u_{i}>u_{i+1}\end{cases}
$$

Then

$$
\varphi\left(e_{z}\right)(v, v)=\varphi\left(e_{z}\right)\left(u_{0}, u_{0}\right)+\sum_{i=0}^{m-1} \Delta_{\Gamma, i}(z)
$$

where $\Delta_{\Gamma, i}(z)=\varphi\left(e_{z}\right)\left(u_{i+1}, u_{i+1}\right)-\varphi\left(e_{z}\right)\left(u_{i}, u_{i}\right)$. By Lemma 5.7

$$
\Delta_{\Gamma, i}(z)= \begin{cases}-1, & \left(u_{i}<u_{i+1} \wedge z=v_{i}\right) \vee\left(u_{i}>u_{i+1} \wedge z=w_{i}\right), \\ 1, & \left(u_{i}<u_{i+1} \wedge z=w_{i}\right) \vee\left(u_{i}>u_{i+1} \wedge z=v_{i}\right), \\ 0, & z \notin\left\{v_{i}, w_{i}\right\}\end{cases}
$$

Since the right-hand side of (11) depends only on $\theta_{\varphi}$ and $\varphi\left(e_{z}\right)\left(u_{0}, u_{0}\right)$, we have $\varphi\left(e_{z}\right)(v, v)=\psi\left(e_{z}\right)(v, v)$. This proves $\varphi\left(e_{z}\right)=\psi\left(e_{z}\right)$, as $\varphi\left(e_{z}\right), \psi\left(e_{z}\right) \in L_{0}$.

Definition 5.9. Let $\theta: B \rightarrow B$ be a bijection and $\Gamma: u_{0}, u_{1}, \ldots, u_{m}=u_{0}$ a closed walk in $X$. We introduce the following 4 functions $X \rightarrow \mathbb{N}$ :

$$
\begin{aligned}
& s_{\theta, \Gamma}^{+}(z)=\mid\left\{i: u_{i}<u_{i+1} \text { and } \exists w>z \text { such that } \theta\left(e_{z w}\right)=e_{u_{i} u_{i+1}}\right\} \mid, \\
& s_{\theta, \Gamma}^{-}(z)=\mid\left\{i: u_{i}>u_{i+1} \text { and } \exists w>z \text { such that } \theta\left(e_{z w}\right)=e_{u_{i+1} u_{i}}\right\} \mid, \\
& t_{\theta, \Gamma}^{+}(z)=\mid\left\{i: u_{i}<u_{i+1} \text { and } \exists w<z \text { such that } \theta\left(e_{w z}\right)=e_{u_{i} u_{i+1}}\right\} \mid, \\
& t_{\theta, \Gamma}^{-}(z)=\mid\left\{i: u_{i}>u_{i+1} \text { and } \exists w<z \text { such that } \theta\left(e_{w z}\right)=e_{u_{i+1} u_{i}}\right\} \mid .
\end{aligned}
$$

Remark 5.10. Let $\varphi \in \widetilde{\operatorname{LAut}}(I(X, K))$ and $\theta=\theta_{\varphi}$. Then for any closed walk $\Gamma: u_{0}, u_{1}, \ldots, u_{m}=u_{0}$ in $X$ and for all $z \in X$

$$
s_{\theta, \Gamma}^{+}(z)-s_{\theta, \Gamma}^{-}(z)=t_{\theta, \Gamma}^{+}(z)-t_{\theta, \Gamma}^{-}(z) .
$$

Indeed, by (8) we have $0=\sum_{i=0}^{m-1}\left(\varphi\left(e_{z}\right)\left(u_{i+1}, u_{i+1}\right)-\varphi\left(e_{z}\right)\left(u_{i}, u_{i}\right)\right)=-s_{\theta, \Gamma}^{+}(z)+$ $s_{\theta, \Gamma}^{-}(z)+t_{\theta, \Gamma}^{+}(z)-t_{\theta, \Gamma}^{-}(z)$.

Definition 5.11. A bijection $\theta: B \rightarrow B$ is said to be admissible if (13) holds for any closed walk $\Gamma: u_{0}, u_{1}, \ldots, u_{m}=u_{0}$ in $X$ and for all $z \in X$. In particular, if $X$ is a tree, then any bijection $\theta: B \rightarrow B$ is admissible.

Example 5.12. Let $X$ be a 2 -crown, i.e., $X=\{1,2,3,4\}$ with the following Hasse diagram.

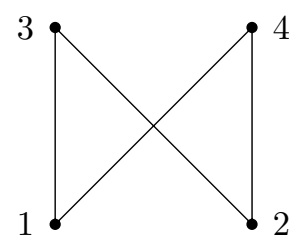


Consider $\theta: B \rightarrow B$ such that $\theta\left(e_{13}\right)=e_{14}, \theta\left(e_{14}\right)=e_{13}, \theta\left(e_{23}\right)=e_{23}$ and $\theta\left(e_{24}\right)=e_{24}$. Then $\theta$ is not admissible. Indeed, for the cycle $\Gamma: 1<3>2<4>1$ we have $s_{\theta, \Gamma}^{+}(3)=s_{\theta, \Gamma}^{-}(3)=t_{\theta, \Gamma}^{+}(3)=0$ and $t_{\theta, \Gamma}^{-}(3)=2$, so that $s_{\theta, \Gamma}^{+}(3)-s_{\theta, \Gamma}^{-}(3) \neq$ $t_{\theta, \Gamma}^{+}(3)-t_{\theta, \Gamma}^{-}(3)$. Observe, however, that $s_{\theta, \Gamma}^{+}(1)=s_{\theta, \Gamma}^{-}(1)=1$ and $t_{\theta, \Gamma}^{+}(1)=$ $t_{\theta, \Gamma}^{-}(1)=0$, so (13) is not invariant under the choice of $z \in X$.

Lemma 5.13. In Definition 5.11 it suffices to verify (13) for any cycle $\Gamma$ in $X$.

Proof. Let us fix a bijection $\theta: B \rightarrow B$ and $z \in X$. Assume that (13) is valid for any cycle. We will prove that it also holds for any closed walk $\Gamma: u_{0}, u_{1}, \ldots, u_{m}=u_{0}$. We call a pair of indices $(i, j), i<j-1$, a repetition if $u_{i}=u_{j}$. The proof will be by induction on the number of repetitions in $\Gamma$. If $\Gamma$ has only one repetition $(0, m)$ and $m \geq 4$, then it is a cycle, so (13) holds by assumption. The case $m=3$ is impossible, since the Hasse diagram of a poset has no triangles. If $m=2$, then $\Gamma$ is of the form $x<y>x$ or $x>y<x$. In this case it always satisfies (13). Indeed, let $\Gamma: x<y>x$. If $\theta\left(e_{z w}\right)=e_{x y}$ for some $w>z$, then $s_{\theta, \Gamma}^{ \pm}(z)=1$ and $t_{\theta, \Gamma}^{ \pm}(z)=0$; and if $\theta\left(e_{w z}\right)=e_{x y}$ for some $w<z$, then $s_{\theta, \Gamma}^{ \pm}(z)=0$ and $t_{\theta, \Gamma}^{ \pm}(z)=1$; otherwise $s_{\theta, \Gamma}^{ \pm}(z)=t_{\theta, \Gamma}^{ \pm}(z)=0$. The case $\Gamma: x>y<x$ is analogous.

Now suppose that $\Gamma$ has a repetition different from $(0, m)$, say $(i, j), i<j-1$. If $i>0$ and $j<m$, then $\Gamma$ splits into two closed walks $\Gamma^{\prime}: u_{0}, \ldots, u_{i}, u_{j+1}, \ldots, u_{m}$ and $\Gamma^{\prime \prime}: u_{i}, \ldots, u_{j}$ whose numbers of repetitions are strictly less than that of $\Gamma$. Hence, by induction hypothesis, $\Gamma^{\prime}$ and $\Gamma^{\prime \prime}$ satisfy (13). Since, for any $0 \leq i<m$, either $u_{i}, u_{i+1} \in \Gamma^{\prime}$ or $u_{i}, u_{i+1} \in \Gamma^{\prime \prime}$, then $s_{\theta, \Gamma}^{ \pm}(z)=s_{\theta, \Gamma^{\prime}}^{ \pm}(z)+s_{\theta, \Gamma^{\prime \prime}}^{ \pm}(z)$ and $t_{\theta, \Gamma}^{ \pm}(z)=$ $t_{\theta, \Gamma^{\prime}}^{ \pm}(z)+t_{\theta, \Gamma^{\prime \prime}}^{ \pm}(z)$, so (13) for $\Gamma$ follows from (13) for $\Gamma^{\prime}$ and $\Gamma^{\prime \prime}$. If $i=0$, then $j<m-1$, and we apply the same argument to $\Gamma^{\prime}: u_{0}, \ldots, u_{j}$ and $\Gamma^{\prime \prime}: u_{j}, \ldots, u_{m}$. The case $j=m$ and $i>1$ is similar.

Remark 5.14. Let $\varphi \in \widetilde{\operatorname{LAut}}(I(X, K))$ and $u, v \in X$. Then $\sum_{z \in X} \varphi\left(e_{z}\right)(u, u)=$ $\sum_{z \in X} \varphi\left(e_{z}\right)(v, v) \neq 0$.

Indeed, observe that $\sum_{z \in X} e_{z}=\delta$, so that $\sum_{z \in X} \varphi\left(e_{z}\right)=\varphi(\delta)$. Since $\delta$ is central, so is $\varphi(\delta)$, and it is obviously non-zero, therefore $\varphi(\delta)=k \delta$ for some $k \in K^{*}$, by [16, Corollary 1.3.15].

Definition 5.15. Let $\theta: B \rightarrow B$ be an admissible bijection and $\sigma: X_{<}^{2} \rightarrow K^{*}$ compatible with $\theta$. We say that $\varphi \in \widetilde{\operatorname{LAut}}(I(X, K))$ induces the pair $(\theta, \sigma)$ if (6) holds for all $x<y$.

Lemma 5.16. Let $\theta: B \rightarrow B$ be an admissible bijection which is monotone on maximal chains in $X$ and $\sigma: X_{<}^{2} \rightarrow K^{*}$ compatible with $\theta$. Then there exists $\varphi \in \widetilde{\operatorname{LAut}}(I(X, K))$ inducing $(\theta, \sigma)$.

Proof. We first show that equality (6) defines a Lie automorphism of $J(I(X, K))$. Let $x, y, z, w \in X$ such that $x<y$ and $z<w$. If $x \neq w$ and $y \neq z$, then $\varphi\left(\left[e_{x y}, e_{z w}\right]\right)=\varphi(0)=0$. Moreover, if $\theta\left(e_{x y}\right)=e_{u v}$ and $\theta\left(e_{z w}\right)=e_{s t}$, then $u \neq t$ and $v \neq s$ by Remark 5.6. Thus

$$
\left[\varphi\left(e_{x y}\right), \varphi\left(e_{z w}\right)\right]=\sigma(x, y) \sigma(z, w)\left[\theta\left(e_{x y}\right), \theta\left(e_{z w}\right)\right]=\sigma(x, y) \sigma(z, w)\left[e_{u v}, e_{s t}\right]=0 .
$$

If $y=z$, then $\varphi\left(\left[e_{x y}, e_{y w}\right]\right)=\varphi\left(e_{x w}\right)$. On the other hand,

$$
\begin{aligned}
{\left[\varphi\left(e_{x y}\right), \varphi\left(e_{y w}\right)\right] } & =\sigma(x, y) \sigma(y, w)\left[\theta\left(e_{x y}\right), \theta\left(e_{y w}\right)\right] \\
& =\sigma(x, y) \sigma(y, w)\left(\theta\left(e_{x y}\right) \theta\left(e_{y w}\right)-\theta\left(e_{y w}\right) \theta\left(e_{x y}\right)\right) .
\end{aligned}
$$


If $\theta$ is increasing on a maximal chain containing $x<y<w$, then

$$
\left[\varphi\left(e_{x y}\right), \varphi\left(e_{y w}\right)\right]=\sigma(x, w) \theta\left(e_{x w}\right)=\varphi\left(e_{x w}\right) .
$$

And if $\theta$ is decreasing on a maximal chain containing $x<y<w$, then

$$
\left[\varphi\left(e_{x y}\right), \varphi\left(e_{y w}\right)\right]=-\sigma(x, w)\left(-\theta\left(e_{x w}\right)\right)=\varphi\left(e_{x w}\right) .
$$

Analogously, if $x=w$ we have $\varphi\left(\left[e_{x y}, e_{z x}\right]\right)=-\varphi\left(e_{z y}\right)=\left[\varphi\left(e_{x y}\right), \varphi\left(e_{z x}\right)\right]$. Thus $\varphi$ is a Lie endomorphism of $J(I(X, K))$. Let $e_{u v} \in J(I(X, K))$. There exist unique $x<y$ in $X$ such that $\theta\left(e_{x y}\right)=e_{u v}$. Then $e_{u v}=\sigma(x, y)^{-1} \varphi\left(e_{x y}\right)=\varphi\left(\sigma(x, y)^{-1} e_{x y}\right)$ and so $\varphi$ is bijective.

Now, we need to show that $\varphi$ extends to a Lie automorphism of $I(X, K)$. Let us fix $u_{0} \in X$ and $\varphi\left(e_{z}\right)\left(u_{0}, u_{0}\right) \in K(z \in X)$ in a way that $\sum_{z \in X} \varphi\left(e_{z}\right)\left(u_{0}, u_{0}\right) \neq 0$. Given $v \in X$, there is a walk $\Gamma: u_{0}, u_{1}, \ldots, u_{m}=v$ from $u_{0}$ to $v$, since $X$ is connected. Then we define $\varphi\left(e_{z}\right)(v, v)$ by formulas (11) and (12). We will show that the definition does not depend on the choice of a walk from $u_{0}$ to $v$. For, if there is another walk $\Gamma^{\prime}: u_{0}^{\prime}=u_{0}, u_{1}^{\prime}, \ldots, u_{m^{\prime}}^{\prime}=v$ from $u_{0}$ to $v$, then there is a closed walk $\Omega: u_{0}, u_{1}, \ldots, u_{m}=v=u_{m^{\prime}}^{\prime}, u_{m+1}=u_{m^{\prime}-1}^{\prime}, \ldots, u_{m+m^{\prime}}=u_{0}^{\prime}=u_{0}$. Since $\theta$ is admissible,

$$
\begin{aligned}
0 & =-s_{\theta, \Omega}^{+}(z)+s_{\theta, \Omega}^{-}(z)+t_{\theta, \Omega}^{+}(z)-t_{\theta, \Omega}^{-}(z) \\
& =\sum_{i=0}^{m+m^{\prime}-1} \Delta_{\Omega, i}(z)=\sum_{i=0}^{m-1} \Delta_{\Gamma, i}(z)+\sum_{i=m}^{m+m^{\prime}-1} \Delta_{\Omega, i}(z),
\end{aligned}
$$

where $\Delta_{\Omega, i}(z)$ and $\Delta_{\Gamma, i}(z)$ are given by (12). Hence

$$
\begin{aligned}
\sum_{i=0}^{m-1} \Delta_{\Gamma, i}(z) & =-\sum_{i=m}^{m+m^{\prime}-1} \Delta_{\Omega, i}(z)=\sum_{i=0}^{m^{\prime}-1}\left(-\Delta_{\Omega, i+m}(z)\right) \\
& =\sum_{i=0}^{m^{\prime}-1} \Delta_{\Gamma^{\prime}, m^{\prime}-i-1}(z)=\sum_{i=0}^{m^{\prime}-1} \Delta_{\Gamma^{\prime}, i}(z) .
\end{aligned}
$$

So, $\varphi\left(e_{z}\right)(v, v)$ is well-defined by (11).

Observe that, by the construction, $\varphi$ satisfies (8) for all $u<v$. Indeed, there is a walk starting at $u_{0}$ of the form $\Gamma: u_{0}, \ldots, u_{l}=u, \ldots, u_{m}=v$, where $u_{l}<\cdots<u_{m}$. Using this walk to define $\varphi\left(e_{z}\right)(u, u)$ and $\varphi\left(e_{z}\right)(v, v)$, by (11) we have

$$
\varphi\left(e_{z}\right)(v, v)-\varphi\left(e_{z}\right)(u, u)=\sum_{i=l}^{m-1} \Delta_{\Gamma, i}(z) .
$$

Assume that $\theta^{-1}$ is increasing on a maximal chain containing $u_{l}<\cdots<u_{m}$. Then there exist $v_{l}<\cdots<v_{m}$ such that $\theta\left(e_{v_{i} v_{j}}\right)=e_{u_{i} u_{j}}, l \leq i<j \leq m$. If $z=v_{l}$, then $\Delta_{\Gamma, l}(z)=-1$ and $\Delta_{\Gamma, i}(z)=0$ for all $l<i \leq m-1$ by (12). Hence, the righthand side of (14) equals -1 . But $\theta\left(e_{z v_{m}}\right)=e_{u_{l} u_{m}}=e_{u v}$, so (8) holds for $u<v$. Similarly, if $z=v_{m}$, then $\Delta_{\Gamma, m-1}(z)=1$ and $\Delta_{\Gamma, i}(z)=0$ for all $l \leq i<m-1$ by (12). Hence, the right-hand side of (14) equals 1 and $\theta\left(e_{v_{l} z}\right)=e_{u v}$ justifying (8) for $u<v$. If $z=v_{k}$ with $l<k<m$, then $\Delta_{\Gamma, k}(z)=-1, \Delta_{\Gamma, k-1}(z)=1$ and $\Delta_{\Gamma, i}(z)=0$ for all $l \leq i \leq m-1, i \notin\{k-1, k\}$. Hence, the right-hand side of (14) equals 0 and $\theta\left(e_{v_{l} v_{m}}\right)=e_{u v}$ with $z \notin\left\{v_{l}, v_{m}\right\}$, so (8) still holds. Finally, if $z \neq v_{i}$ for all $l \leq i \leq m$, then $\Delta_{\Gamma, i}(z)=0$ for all $l \leq i \leq m-1$, so that the right-hand side of (14) again equals 0 . The decreasing case is similar. 
Now, define $\varphi\left(e_{z}\right)=\sum_{v \in X} \varphi\left(e_{z}\right)(v, v) e_{v} \in D(X, K)$. Automatically, we have $\left[\varphi\left(e_{x}\right), \varphi\left(e_{y}\right)\right]=0$ for all $x, y$. Let $x<y$ and $\theta\left(e_{x y}\right)=e_{u v}$. Then it follows from (8) that for all $z \in X$,

$$
\begin{aligned}
{\left[\varphi\left(e_{z}\right), \varphi\left(e_{x y}\right)\right] } & =\left[\varphi\left(e_{z}\right)_{D}, \sigma(x, y) e_{u v}\right]=\sigma(x, y)\left(\varphi\left(e_{z}\right)(u, u)-\varphi\left(e_{z}\right)(v, v)\right) e_{u v} \\
& =\left\{\begin{array}{ll}
\sigma(x, y) e_{u v}, & z=x, \\
-\sigma(x, y) e_{u v}, & z=y, \\
0, & z \notin\{x, y\}
\end{array}= \begin{cases}\varphi\left(e_{x y}\right), & z=x, \\
-\varphi\left(e_{x y}\right), & z=y, \\
0, & z \notin\{x, y\}\end{cases} \right. \\
& =\varphi\left(\left[e_{z}, e_{x y}\right]\right) .
\end{aligned}
$$

Thus, the extended $\varphi$ is a Lie endomorphism of $I(X, K)$.

It remains to prove that $\varphi$ is bijective. Since $\varphi$ maps bijectively $J(I(X, K))$ onto itself and $\varphi(D(X, K)) \subseteq D(X, K)$ by construction, then it suffices to show that $\varphi$ is bijective on $D(X, K)$. Suppose that $\sum_{z \in X} k_{z} \varphi\left(e_{z}\right)=0$ for some $\left\{k_{z}\right\}_{z \in X} \subseteq K$, at least one of which is non-zero. Let $x<y$ and $\theta\left(e_{x y}\right)=e_{u v}$. Then

$$
\begin{aligned}
\sum_{z \in X} k_{z}\left(\varphi\left(e_{z}\right)(v, v)-\varphi\left(e_{z}\right)(u, u)\right) & =\left(\sum_{z \in X} k_{z} \varphi\left(e_{z}\right)\right)(v, v)-\left(\sum_{z \in X} k_{z} \varphi\left(e_{z}\right)\right)(u, u) \\
& =0 .
\end{aligned}
$$

On the other hand, by (8) the left-hand side of (15) equals $k_{y}-k_{x}$. Thus, $k_{x}=k_{y}$. Since $X$ is connected, we have $k_{x}=k_{y} \neq 0$ for all $x, y \in X$, whence

$$
0=\sum_{z \in X} k_{z} \varphi\left(e_{z}\right)\left(u_{0}, u_{0}\right)=k_{u_{0}} \sum_{z \in X} \varphi\left(e_{z}\right)\left(u_{0}, u_{0}\right),
$$

which contradicts the choice of $\varphi\left(e_{z}\right)\left(u_{0}, u_{0}\right)$ at the beginning of the proof. Therefore $\varphi$ is a Lie automorphism of $I(X, K)$. Moreover, since $\varphi\left(L_{i}\right) \subseteq L_{i}$ for all $i \geq 0$, then $\varphi \in \widetilde{\operatorname{LAut}}(I(X, K))$, by Remark 4.8 .

Definition 5.17. Let $X=\left\{x_{1}, \ldots, x_{n}\right\}$. Given an admissible bijection $\theta: B \rightarrow B$ which is monotone on maximal chains in $X$, a map $\sigma: X_{<}^{2} \rightarrow K^{*}$ compatible with $\theta$ and a sequence $c=\left(c_{1}, \ldots, c_{n}\right) \in K^{n}$ such that $\sum_{i=1}^{n} c_{i} \in K^{*}$, define $\tau=\tau_{\theta, \sigma, c}$ to be the elementary Lie automorphism of $I(X, K)$ such that $\left.\tau\right|_{J(I(X, K))}$ is given by the right-hand side of (6) and $\left.\tau\right|_{D(X, K)}$ is determined by

$$
\tau\left(e_{x_{i}}\right)\left(x_{1}, x_{1}\right)=c_{i},
$$

$i=1, \ldots, n$, as in Lemmas 5.8 and 5.16

Theorem 5.18. Each $\varphi \in \widetilde{\operatorname{LAut}}(I(X, K))$ can be uniquely represented in the form

$$
\varphi=\tau_{\theta, \sigma, c}
$$

where $\theta: B \rightarrow B$ is an admissible bijection which is monotone on maximal chains in $X, \sigma: X_{<}^{2} \rightarrow K^{*}$ is a map compatible with $\theta$ and $c=\left(c_{1}, \ldots, c_{n}\right) \in K^{n}$ is such that $\sum_{i=1}^{n} c_{i} \in K^{*}$.

Proof. Let $X=\left\{x_{1}, \ldots, x_{n}\right\}$. We define $\theta=\theta_{\varphi}, \sigma=\sigma_{\varphi}$ and $c_{i}=\varphi\left(e_{x_{i}}\right)\left(x_{1}, x_{1}\right)$, $i=1, \ldots, n$. Then $\theta, \varphi$ and $c$ satisfy the desired properties by Lemmas 5.2 and 5.4 and Remarks 5.10 and 5.14. Hence, $\tau:=\tau_{\theta_{\varphi}, \sigma_{\varphi}, c}$ is well-defined. For all $x_{i}<$ $x_{j}$ we have $\varphi\left(e_{x_{i} x_{j}}\right)=\sigma\left(x_{i}, x_{j}\right) \theta\left(e_{x_{i} x_{j}}\right)=\tau\left(e_{x_{i} x_{j}}\right)$, so $\left.\varphi\right|_{J(I(X, K))}=\left.\tau\right|_{J(I(X, K))}$. Moreover, $\left.\varphi\right|_{D(X, K)}=\left.\tau\right|_{D(X, K)}$ by Lemma [5.8. The uniqueness of (17) follows from (6) and (16). 
Corollary 5.19. Each Lie automorphism $\varphi$ of $T_{n}(K)$ is a composition $\psi \circ \mu$, where $\psi$ is an automorphism or the negative of an anti-automorphism and $\mu$ is a Lie automorphism given by $\mu\left(e_{i j}\right)=e_{i j}$ for all $i<j$ and $\mu\left(e_{i}\right)=e_{i}+\alpha_{i} \delta$ for all $i$, where $1+\sum_{i=1}^{n} \alpha_{i} \in K^{*}$. Equivalently, $\varphi=\psi+\nu$, where $\nu\left(e_{i j}\right)=0$ for all $i<j$ and $\nu\left(e_{i}\right)=\alpha_{i} \delta$ for all $i$, therefore $\varphi$ is proper.

Proof. Indeed, $T_{n}(K) \cong I(X, K)$ where $X$ is the chain $x_{1}=1<2<\cdots<$ $n=x_{n}$. Let $\varphi \in \operatorname{LAut}(I(X, K))$. In view of Theorem 4.15 we may assume that $\varphi \in \widetilde{\operatorname{LAut}}(I(X, K))$. Then $\varphi=\tau_{\theta, \sigma, c}$ as in Theorem 5.18. The bijection $\theta: B \rightarrow B$ is increasing on $X$ if and only if $\theta=\mathrm{id}_{B}$. In this case $\varphi\left(e_{i j}\right)=\sigma(i, j) e_{i j}, i<j$, for some $\sigma: X_{<}^{2} \rightarrow K^{*}$ satisfying $\sigma(i, k)=\sigma(i, j) \sigma(j, k), i<j<k$. Observe that $\sigma(i, j)=\sigma(1, i)^{-1} \sigma(1, j), 1<i<j$, so $\varphi\left(e_{i j}\right)=\eta^{-1} e_{i j} \eta, i<j$, where $\eta=e_{1}+\sum_{i=2}^{n} \sigma(1, i) e_{i}$. Moreover, $\eta^{-1} e_{i} \eta=e_{i}$ for any $i$. It also follows from (11) and (12) that $\varphi\left(e_{i}\right)(j, j)=c_{i}+\delta_{i j}$ for all $i \neq 1$ and $\varphi\left(e_{1}\right)(j, j)=c_{1}-1+\delta_{1 j}$, whence $\varphi\left(e_{i}\right)=e_{i}+\alpha_{i} \delta$, where $\alpha_{1}=c_{1}-1$ and $\alpha_{i}=c_{i}, i>1$. The decompositions of $\varphi$ as $\psi \circ \mu$ and $\psi+\nu$, in which $\psi$ is the conjugation by $\eta^{-1}$, are now clear.

If $\theta$ is decreasing on $X$, then $\theta\left(e_{i j}\right)=e_{n-j+1, n-i+1}, 1 \leq i<j \leq n$, so $\varphi\left(e_{i j}\right)=$ $\sigma(i, j) e_{n-j+1, n-i+1}$, where $\sigma: X_{<}^{2} \rightarrow K^{*}$ is such that $\sigma(i, k)=-\sigma(i, j) \sigma(j, k)$, $i<j<k$. Therefore, $\varphi=\lambda \circ \varphi^{\prime}$, where $\lambda\left(e_{i j}\right)=-e_{n-j+1, n-i+1}$ is the negative of an anti-automorphism of $I(X, K)$ and $\varphi^{\prime}$ is as in the increasing case.

Example 5.20. Let $X=\{1,2,3,4\}$, where $1<2<3,1<4$ and 4 is incomparable with 2 and 3 . Observe that $I(X, K)$ has no anti-automorphism by 4 , Theorem 3]. Consider the following linear map: $\varphi\left(e_{1}\right)=-e_{3}-e_{4}, \varphi\left(e_{12}\right)=e_{23}, \varphi\left(e_{13}\right)=$ $-e_{13}, \varphi\left(e_{14}\right)=e_{14}, \varphi\left(e_{2}\right)=e_{1}+e_{3}+e_{4}, \varphi\left(e_{23}\right)=e_{12}, \varphi\left(e_{3}\right)=e_{2}+e_{3}, \varphi\left(e_{4}\right)=e_{4}$. Then $\varphi=\tau_{\theta, \sigma, c}$, where $\theta\left(e_{12}\right)=e_{23}, \theta\left(e_{23}\right)=e_{12}, \theta\left(e_{13}\right)=e_{13}, \theta\left(e_{14}\right)=e_{14}$, $\sigma(1,2)=\sigma(2,3)=\sigma(1,4)=1, \sigma(1,3)=-1, c_{1}=c_{3}=c_{4}=0, c_{2}=1$. Assume that $\varphi$ is proper, i.e., $\varphi=\psi+\nu$, where $\psi \in \operatorname{Aut}(I(X, K))$ and $\nu$ is a central-valued map. There is $i \in X$ such that $\psi\left(e_{1}\right)_{D}=e_{i}$ by [10, Lemma 1]. Then $\varphi\left(e_{1}\right)_{D}=e_{i}+\alpha \delta$ for some $\alpha \in K$, i.e., $\varphi\left(e_{1}\right)(i, i)=\varphi\left(e_{1}\right)(j, j)+1$ for all $j \neq i$, a contradiction.

\section{ACKNOWLEDGEMENTS}

This work was partially supported by CNPq 404649/2018-1 and by the Fundação para a Ciência e a Tecnologia (Portuguese Foundation for Science and Technology) through the project PTDC/MAT-PUR/31174/2017. We are grateful to the referee for pointing out a gap in the proof of Proposition 4.5, as well as numerous small inaccuracies throughout the text. In particular, we thank the referee for the suggestion to reduce the proof of Lemma 3.7.

\section{REFERENCES}

[1] Akkurt, E., Akkurt, M., and Barker, G. P. Jordan homomorphisms of the structural matrix algebras. Linear Multilinear Algebra 63, 12 (2015), 2518-2525.

[2] Brusamarello, R., Fornaroli, E. Z., and Khrypchenko, M. Jordan isomorphisms of finitary incidence algebras. Linear Multilinear Algebra 66, 3 (2018), 565-579.

[3] Brusamarello, R., Fornaroli, E. Z., and Khrypchenko, M. Jordan isomorphisms of the finitary incidence ring of a partially ordered category. Colloq. Math. 159, 2 (2020), 285-307.

[4] Brusamarello, R., Fornaroli, E. Z., and Santulo Jr, E. A. Anti-automorphisms and involutions on (finitary) incidence algebras. Linear Multilinear Algebra 60, 2 (2012), 181-188.

[5] CAO, Y. Automorphisms of certain Lie algebras of upper triangular matrices over a commutative ring. J. Algebra 189, 2 (1997), 506-513. 
[6] Cecil, A. J. Lie isomorphisms of triangular and block-triangular matrix algebras over commutative rings. Master's thesis, University of Victoria, 2016.

[7] Đoković, D. Ž. Automorphisms of the Lie algebra of upper triangular matrices over a connected commutative ring. J. Algebra 170, 1 (1994), 101-110.

[8] Doubilet, P., Rota, G.-C., and Stanley, R. P. On the foundations of combinatorial theory. VI. The idea of generating function. In Proceedings of the Sixth Berkeley Symposium on Mathematical Statistics and Probability, vol. II: Probability theory. Univ. California Press, 1972, pp. 267-318.

[9] Hua, L. K. A theorem on matrices over a sfield and its applications. J. Chinese Math. Soc. (N.S.) 1 (1951), 110-163.

[10] Khripchenko, N. S. Automorphisms of finitary incidence rings. Algebra and Discrete Math. 9, 2 (2010), 78-97.

[11] Khrypchenko, M., and Wei, F. Lie-type Derivations of Finitary Incidence Algebras. Rocky Mountain J. Math. 50, 1 (2020), 163-175.

[12] Marcoux, L. W., And Sourour, A. R. Commutativity preserving linear maps and Lie automorphisms of triangular matrix algebras. Linear Algebra Appl. 288, 1-3 (1999), 89-104.

[13] Martindale 3rd, W. S. Lie isomorphisms of primitive rings. Proc. Amer. Math. Soc. 14 (1963), 909-916.

[14] Martindale 3rd, W. S. Lie isomorphisms of prime rings. Trans. Amer. Math. Soc. 142 (1969), 437-455.

[15] Martindale 3Rd, W. S. Lie isomorphisms of simple rings. J. Lond. Math. Soc. 44 (1969), 213-221.

[16] Spiegel, E., and O’Donnell, C. J. Incidence Algebras. New York, NY: Marcel Dekker, 1997.

[17] Wang, D., And Xiao, Z. Lie triple derivations of incidence algebras. Comm. Algebra 47, 5 (2019), 1841-1852.

[18] XiaO, Zh. ANd YANG, Yu. Lie n-derivations of incidence algebras. Comm. Algebra 48, 1 (2020), 105-118.

[19] Zhang, X., and Khrypchenko, M. Lie derivations of incidence algebras. Linear Algebra Appl. 513 (2017), 69-83.

Departamento de Matemática, Universidade Estadual de Maringá, Maringá, PR, CEP: 87020-900, BRAZIL

Email address: ezancanella@uem.br

Departamento de Matemática, Universidade Federal de Santa Catarina, Campus Reitor João David Ferreira Lima, Florianópolis, SC, CEP: 88040-900, Brazil

Email address: nskhripchenko@gmail.com

Departamento de Matemática, Universidade Estadual de Maringá, Maringá, PR, CEP: 87020-900, BRAZIL

Email address: easjunior@uem.br 